\title{
ON THE GAUSS MAP OF MINIMAL SURFACES WITH FINITE TOTAL CURVATURE
}

\author{
MiN Ru
}

\begin{abstract}
We prove that if a nonflat complete regular minimal surface immersed in $R^{n}$ is of finite total curvature, then its Gauss map can omit at most $(n-1)(n+2) / 2$ hyperplanes in general position in $P^{n-1}(\mathbb{C})$.
\end{abstract}

\section{INTRODUCTION}

There have been several results devoted to studying the "value distribution" properties of the Gauss map of a nonflat complete regular minimal surface. In [3], Fujimoto proved that the Gauss map of a nonflat complete regular minimal surface immersed in $R^{3}$ can omit at most four points of the sphere. With the additional condition of finite total curvature, Osserman [6] showed that the Gauss map can omit at most three points of the sphere. For the Gauss map of a nonflat complete regular minimal surface immersed in $R^{n}$, the author [7] has shown that it can omit at most $n(n+1) / 2$ hyperplanes in general position in $P^{n-1}(\mathbb{C})$, while the "nondegenerate of the Gauss map" case is due to Fujimoto (see [4]). The purpose of this paper is to improve on a theorem of Chern and Osserman [2]. We shall show that the nondegenerate condition in the Theorem 4 of [2] can be removed by using the powerful tool called "Nochka weights". The theorem will be stated in Section 2 .

\section{FaCts Concerning Minimal SURfaces in $R^{n}$ and the STATEMENT OF RESULTS}

We shall recall some basic facts concerning minimal surfaces immersed in $R^{n}$. For further details, we refer to Chern and Osserman [2].

Let $S_{0}$ be a Riemann surface and let $\alpha_{1}, \ldots, \alpha_{n}$ be analytic differentials on $S_{0}$, which we assume to be not all identically zero. Suppose that in terms of a local parameter $\zeta$, we have $\alpha_{k}=\phi_{k} d \zeta, 1 \leqslant k \leqslant n$. Then under the condition

$$
\sum_{1 \leqslant k \leqslant n} \phi_{k}^{2} \equiv 0
$$

Received 20 September 1990

Copyright Clearance Centre, Inc. Serial-fee code: 0004-9729/91 \$A2.00+0.00. 
the surface $x(p): S_{0} \rightarrow R^{n}$, defined by

$$
x_{k}=\operatorname{Re} \int \alpha_{k}
$$

is called a generalised minimal surface assuming the integrals to have single-valued real parts. If furthermore

$$
\sum_{1 \leqslant k \leqslant n}\left|\phi_{k}\right|^{2} \neq 0
$$

then the surface is called a regular minimal surface.

If we set $\zeta=\xi_{1}+i \xi_{2}$, then we have

$$
\phi_{k}=\frac{\partial x_{k}}{\partial \xi_{1}}-i \frac{\partial x_{k}}{\partial \xi_{2}}
$$

and if we denote by

$$
g_{i j}=\frac{\partial x}{\partial \xi_{i}} \cdot \frac{\partial x}{\partial \xi_{j}} \quad 1 \leqslant i, j \leqslant 2
$$

the coefficients of the first fundamental form of the surface (2.2), then condition (2.1) becomes

$$
g_{11}-g_{22}-2 i g_{12}=0
$$

meaning that $\xi_{1}, \xi_{2}$ are isothermal parameters. This condition may also be written in the form

$$
g_{i j}=\lambda^{2} \delta_{i j} \quad \lambda=\lambda(\zeta)
$$

where

$$
\lambda^{2}=\left|\frac{\partial x}{\partial \xi_{1}}\right|^{2}=\left|\frac{\partial x}{\partial \xi_{2}}\right|^{2}=\frac{1}{2} \sum_{1 \leqslant k \leqslant n}\left|\phi_{k}\right|^{2} .
$$

The Gaussian curvature is given by

$$
k=-\frac{\Delta \log \lambda}{\lambda^{2}}=-\frac{4\left|\phi \wedge \phi^{\prime}\right|^{2}}{|\phi|^{6}}
$$

where

$$
\phi=\left(\phi_{1}, \ldots, \phi_{n}\right), \quad|\phi|^{2}=\sum_{1 \leqslant k \leqslant n}\left|\phi_{k}\right|^{2}
$$

and

$$
\left|\phi \wedge \phi^{\prime}\right|^{2}=\sum_{1 \leqslant k \leqslant n}\left|\phi_{j} \phi_{k}^{\prime}-\phi_{j}^{\prime} \phi_{k}\right|^{2} .
$$


Let $D$ be a domain in the $\zeta$-plane, and denote by $S$ the corresponding part of the surface (2.2). Then the area of $S$ is

$$
A(S)=\iint_{D} \lambda^{2} d \xi_{1} d \xi_{2}=\frac{1}{2} \iint_{D}|\phi|^{2} d \xi_{1} d \xi_{2}
$$

and the total curvature of $S$ is

$$
C(S)=\iint_{D} k d A=\iint_{D} k \lambda^{2} d \xi_{1} d \xi_{2}=-2 \iint_{D} \frac{\left|\phi \wedge \phi^{\prime}\right|^{2}}{|\phi|^{4}} d \xi_{1} d \xi_{2} .
$$

The generalised Gauss map is the map

$$
G: S_{0} \rightarrow Q_{n-2} \subset P^{n-1}(\mathbb{C})
$$

with homogeneous coordinates $\overline{\phi_{1}(\zeta)}, \ldots, \overline{\phi_{n}(\zeta)}$, where

$$
Q_{n-1}=\left\{\left[z_{1}: \ldots: z_{n}\right] \subset P^{n-1}(\mathbb{C}) \mid z_{1}^{2}+\ldots+z_{n}^{2}=0\right\}
$$

We have the following theorem:

MaIN Theorem. Let $S$ be a nonflat complete regular minimal surface in $R^{n}$ defined by a map (2.2) on a Riemann surface $S_{0}$, with the Gauss map defined by (2.14). Suppose that $S$ is of finite total curvature. Then $G_{0}\left(S_{0}\right)$ can fail to intersect at most $(n-1)(n+2) / 2$ hyperplanes in general position in $P^{n-1}(\mathbb{C})$.

\section{Plüker formulas for algebraic curVe and NochKa weights for HYPERPLANES IN SUBGENERAL POSITION}

\section{(A) PlüKer Formulas.}

We state the Plüker formulas for an algebraic curve in a complex projective space; see [8], pp.41-65. Let $W$ be a compact Riemann surface of genus $g$ and let $f: W \rightarrow$ $P^{m}(\mathbb{C})$ be a nondegenerate algebraic curve (that is, $f(W)$ is not contained in any hyperplane in $P^{m}(\mathbb{C})$ ). For a suitable choice of homogeneous coordinates $\zeta_{0}, \ldots, \zeta_{m}$ in $P^{m}(\mathbb{C})$, the equations of the curve can be put locally into the normal form

$$
\begin{gathered}
\zeta_{0}=t^{\zeta_{0}}+\ldots \\
\cdots \\
\zeta_{m}=t^{\delta_{m}}+\ldots
\end{gathered}
$$

where

$$
0=\delta_{0}<\delta_{1}<\ldots<\delta_{m},
$$


and where $t$ is a local parameter on $W$. The integers

$$
\nu_{k}=\delta_{k+1}-\delta_{k}-1 \quad 0 \leqslant k \leqslant m-1
$$

are called the stationary indices of order $k$ at the point $t=0$. The stationary point, that is, points with non-zero stationary index, are isolated and hence are finite in number. We will denote by $\sigma_{k}$ the sum of all stationary indices of order $k$. Let $d_{k}$, $0 \leqslant k \leqslant m-1$, be the order of rank $k$ of the algebraic curve; geometrically this is the order of the associated curve of rank $k$, that is, the curve formed by the osculating space of dimension $k$. Then Plüker formulas are

$$
\sigma_{k}=2 d_{k}-d_{k+1}-d_{k-1}+2(g-1), \quad 1 \leqslant k \leqslant m-1
$$

with the convention $d_{-1}=d_{m}=0$. From (3.4), it follows that

$$
\sum_{1 \leqslant h \leqslant m}(m+1-h) \sigma_{h-1}=(m+1) d_{0}+m(m+1)(g-1) .
$$

(B) NOCHKa Weights.

We consider $q$ hyperplanes $H_{j}(1 \leqslant j \leqslant q)$ in $P^{m}(\mathbb{C})$ which are given by

$$
H_{j}:\left(A_{j}, W\right)=0 \quad(1 \leqslant j \leqslant q)
$$

for $A_{j} \in C^{m+1}-\{0\}$, where $q>N \geqslant m$ and $(A, W)$ means $a_{0} w_{0}+\ldots+a_{n} w_{n}$ for a vector $A=\left(a_{0}, \ldots, a_{m}\right)$ and homogeneous coordinates $W=\left[w_{0}, \ldots, w_{n}\right]$.

According to Nochka [5] and Chen [1], we give the following definition.

Definition 3.1: We say that hyperplane $H_{1}, \ldots, H_{q}$ are in $N$-subgeneral position if, for every $1 \leqslant j_{0}<\ldots<j_{N} \leqslant q, A_{j_{0}}, \ldots, A_{j_{N}}$ generate $C^{m+1}$. If $N=m$, then we say that $H_{1}, \ldots, H_{q}$ are in general position.

It is easy to check that if $H_{1}, \ldots, H_{q}$ are hyperplanes in general position in $P^{n-1}(\mathbb{C})$, and we embed $P^{k}(\mathbb{C})$ as the subspace of $P^{n-1}(\mathbb{C})$ for $1 \leqslant k<n-1$, then $H_{i} \cap P^{k}(\mathbb{C})(1 \leqslant i \leqslant q)$ are in $(n-1)$-subgeneral position in $P^{k}(\mathbb{C})$.

Nochka [5] and Chen [1] have given the following lemmas to prove Cartan's conjecture.

Lemma 3.2. Let $H_{1}, \ldots, H_{q}$ be hyperplanes in $P^{m}(\mathbb{C})$ located in $N$-subgeneral position, where $q>2 N-m+1$. Then there exists some constants $\omega(1), \ldots, \omega(q)$ and $\theta$ satisfying the following conditions:

(i) $0<\omega(j) \theta \leqslant 1 \quad(1 \leqslant j \leqslant q)$

(ii) $\theta\left(\sum_{j=1}^{q} \omega(j)-m-1\right)=q-2 N+m-1$

(iii) $(N+1) /(m+1) \leqslant \theta \leqslant(2 N-m+1) /(m+1)$. 
For the proof, see [5] or Chen [1, Theorem 0.3]. We call the constants $\omega(j)$ $(1 \leqslant j \leqslant q)$ and $\theta$ in the above lemma the Nochka weights and the Nochka constant for $H_{1}, \ldots, B_{q}$ respectively.

The following lemma is crucial to the proof of the main theorem using Nochka weights.

Lemma 3.3. Let $H_{1}, \ldots, H_{q}$ be hyperplanes in $P^{m}(\mathbb{C})$ located in $N$-subgeneral position, where $q>2 N-m+1$. Let $\omega(j)(1 \leqslant j \leqslant q)$ be their Nochka weights. Take $A \subset\{1,2, \ldots, q\}$, with $0<\# A=N+1$. Let $E_{j}(1 \leqslant j \leqslant q)$ be real numbers with $E_{j} \geqslant 1$. Then there exists a subindex set $\left\{j_{0}, \ldots j_{m}\right\} \subset A$ such that

$$
\prod_{j \in A}\left(E_{j}\right)^{\omega(j)} \leqslant \prod_{i=0}^{m} E_{j i}
$$

and the hyperplanes $H_{j_{0}}, \ldots, H_{j_{m}}$ are in general position in $P^{m}(\mathbb{C})$.

For the proof, see Chen [1, Theorem 1.2].

\section{Some theorems proved by Chern and Osserman}

We recall some theorems proved by Chern and Osserman [2].

Definition 4.1: The Gauss map (2.14) is called algebraic if the surface $S_{0}$ is conformally equivalent to a region $D$ on a compact Riemann surface $W$, and if, when the differentials $\alpha_{k}$ are considered as analytic differentials on $D$, the ratios $\alpha_{k} / \alpha_{m}$ extend to meromorphic functions on $W$, whenever $\alpha_{m} \not \equiv 0$.

THEOREM A. Let $S$ be a minimal surface defined by (2.2) on a Riemann surface $S_{0}$. If $S$ is a complete regular minimal surface, then the following four statements are equivalent:

(a) $S$ has finite total curvature;

(b) there exists an integer $N$ such that the image of $S_{0}$ under the Gauss map intersects at most $N$ times all hyperplanes which do not contain it;

(c) the Gauss map of $S_{0}$ is algebraic;

(d) the surface $S_{0}$ is conformally equivalent to a compact surface $W$ punctured at a finite number of points $p_{1}, \ldots, p_{r}$, and the differentials $\alpha_{k}$ are either regular or have a pole at each $p_{j}$.

For the proof, see [2], Theorem 1.

THEOREM B. Let $S$ be a complete regular minimal surface with Euler characteristic $\chi$ and $r$ boundary components. Then

$$
C(S) \leqslant 2 \pi(\chi-r) \text {. }
$$

For the proof, see [2], Theorem 2. 
THEOREM C. The total curvature of a complete regular minimal surface is either $-\infty$ or $-2 \pi N$ where $N$ is the integer in statement (b) of Theorem $A$.

For the proof, see [2] Corollary.

\section{PRoOF OF THE MAIN THEOREM}

ProOF OF THE MAIN THEOREM: Under the hypotheses of the main theorem, it follows from Theorem A that $S_{0}$ is conformally equivalent to a compact Riemann surface $W$ punctured at the points $p_{j}, 1 \leqslant j \leqslant r$, and that the Gauss map $G$ extends to an antiholomorphic map of $W$ into $P^{n-1}(\mathbb{C})$. Take a number $m, 1 \leqslant m \leqslant n-1$ such that $G(W)$ is contained in $P^{m}(\mathbb{C})$ but none of lower dimension. Then $G$ is a non-degenerate algebraic curve in $P^{m}(\mathbb{C})$. Let $\pi_{i}, 1 \leqslant i \leqslant q$, be hyperplanes in general position in $P^{n-1}(\mathbb{C})$ which do not intersect $G\left(S_{0}\right)$. Let $H_{i}=\pi_{i} \cap P^{m}(\mathbb{C})$. Then $H_{i}, 1 \leqslant i \leqslant q$, are hyperplanes in $P^{m}(\mathbb{C})$ in $(n-1)$-subgeneral position, and do not intersect $G\left(S_{0}\right)$. Then $H_{i}$ intersects $G(W)$ at certain of the points $p_{j}$, with a multiplicity which we denote by $\mu_{i j}$. We have

$$
\sum_{1 \leqslant j \leqslant r} \mu_{i j}=d_{0}
$$

where $d_{0}$ is the order of the algebraic curve $G(W)$.

Because the hyperplanes are in $(n-1)$-subgeneral position in $P^{m}(\mathbb{C})$, at most $n-1$ of the hyperplanes $H_{i}$ can intersect $G(W)$ at $p_{j}$. It follows that there exists a subset $A \subset\{1,2, \ldots, q\}, \# A=n$ such that

$$
\sum_{1 \leqslant i \leqslant q} \omega(i) \mu_{i j} \leqslant \sum_{i \in A} \omega(i) \mu_{i j}
$$

where $\omega(i), 1 \leqslant i \leqslant q$, are Nochka weights for hyperplanes $H_{i}, 1 \leqslant i \leqslant q$.

Applying Lemma 3.3 with $E_{i}=e^{\mu_{i j}}$, it follows that there exists a subindex set $\left\{i_{0}, \ldots, i_{m}\right\} \subset A$ such that

$$
\prod_{i \in A}\left(e^{\mu_{i j}}\right)^{\omega(i)} \leqslant \prod_{k=0}^{m} e^{\mu_{i_{k} j}}
$$

and the $H_{i_{0} j}, \ldots, H_{i_{m} j}$ are hyperplanes in general position in $P^{m}(\mathbb{C})$. Hence

$$
\sum_{i \in A} \omega(i) \mu_{i j} \leqslant \sum_{k=0}^{m} \mu_{i_{k} j}
$$

Since $H_{i_{0}}, \ldots, H_{i_{m} j}$ are in general position in $P^{m}(\mathbb{C})$, we can adopt Chern and Osserman's argument $[2$, p.30]. 
Let (3.1) be the equations of $G(W)$ at $p_{j}$, whose parameter value is $t=0$. At $p_{j}$ the maximum possible value of $\mu_{i_{k} j}$ is $\delta_{m-1}\left(p_{j}\right)$, and that for the unique hyperplane $\zeta_{m-1}=0$. A second hyperplane can intersect $G(W)$ at $p_{j}$ with multiplicity at most $\delta_{m-2}\left(p_{j}\right)$, and a third, if in general position with respect to the first two, at most $\delta_{m-3}\left(p_{j}\right)$, etc. It follows that at most $m$ of the hyperplanes $H_{i_{k} j}, 0 \leqslant k \leqslant m$, can intersect $G(W)$ at $p_{j}$ and we have

$$
\sum_{0 \leqslant k \leqslant m} \mu_{i_{k} j} \leqslant \delta_{1}\left(p_{j}\right)+\ldots+\delta_{m}\left(p_{j}\right)
$$

Combining this with (5.4) and (5.2), we have

$$
\sum_{1 \leqslant i \leqslant q} \omega(i) \mu_{i j} \leqslant \delta_{1}\left(p_{j}\right)+\ldots+\delta_{m}\left(p_{j}\right)
$$

By (3.3) the right side is equal to

$$
\sum_{1 \leqslant h \leqslant m}(m+1-h) \nu_{h-1}\left(p_{j}\right)+\frac{1}{2} m(m+1) .
$$

Combining this with (3.5), (5.1), we get

$$
\sum_{1 \leqslant i \leqslant q} \omega(i) d_{0} \leqslant \sum_{1 \leqslant i \leqslant q} \sum_{1 \leqslant j \leqslant r} \omega(i) \mu_{i j} \leqslant(m+1) d_{0}+\frac{1}{2} m(m+1)\{2(g-1)+r\} .
$$

On the other hand, by Theorem B and Theorem C

$$
d_{0} \geqslant 2(r+g-1)
$$

Eliminating $g$ in the inequalities (5.7) and (5.8), we get

$$
\frac{1}{2} m(m+1) r \leqslant\left\{\frac{1}{2}(m+1)(m+2)-\sum_{1 \leqslant i \leqslant q} \omega(i)\right\} d_{0}
$$

So

$$
\frac{1}{2} m(m+1) r \theta \leqslant\left\{\frac{1}{2}(m+1)(m+2) \theta-\sum_{1 \leqslant i \leqslant q} \theta \omega(i)\right\} d_{0}
$$

where $\theta$ is the Nochka constant. By Lemma 3.2 (ii)

$$
\sum_{1 \leqslant i \leqslant q} \theta \omega(i)=q-2(n-1)+(m-1)+\theta(m+1) .
$$


We have

$$
\begin{aligned}
\frac{1}{2} m(m+1) r \theta & \leqslant\left\{\frac{1}{2}(m+1)(m+2) \theta-q+2(n-1)-m+1-\theta(m+1)\right\} d_{0} \\
& \leqslant\left\{\frac{1}{2} m(m+1) \theta-1+2(n-1)-m+1\right\} d_{0}
\end{aligned}
$$

by Lemma 3.2 (iii) $\theta \leqslant(2(n-1)-m+1) /(m+1)(5.11)$ becomes

$$
\begin{aligned}
\frac{1}{2} m(m+1) r \theta & \leqslant\left\{\left(\frac{1}{2} m+1\right)(2(n-1)-m+1)-q\right\} d_{0} \\
& \leqslant\left\{\frac{1}{2}(2 n-m-1)(m+2)-q\right\} d_{0} .
\end{aligned}
$$

For $1 \leqslant m \leqslant n-1,(2 n-m-1)(m+2) / 2 \leqslant n(n+1) / 2$ therefore

$$
\frac{1}{2} m(m+1) r \theta \leqslant\left\{\frac{1}{2} n(n+1)-q\right\} d_{0} .
$$

Since the left-hand side is strictly positive, this gives

$$
q<\frac{1}{2} n(n+1)
$$

which proves the theorem.

\section{REFERENCES}

[1] W. Chen, 'Defect relations for degenerate meromorphic maps', Trans. Amer. Math. Soc. 319 (1990), 499-515.

[2] S.S. Chern and R. Osserman, 'Complete minimal surfaces in euclidean $n$-space', J. Analyse Math. 19 (1967), 15-34.

[3] H. Fujimoto, 'On the number of exceptional values of the Gauss map of minimal surfaces', J. Math. Soc. Japan 49 (1988), 235-247.

[4] H. Fujimoto, 'Modified defect relations for the Gauss map of minimal surfaces II', J. Differential Geometry 31 (1990), 365-385.

[5] E.I. Nochka, 'On the theory of meromorphic functions', Soviet Math. Dokl. 27 (1983).

[6] R. Osserman, A survey of minimal surfaces, 2nd edition (Dover, New York, 1986).

[7] M. Ru, 'On the Gauss map of minimal surfaces immersed in $R^{n}$,' (preprint) 16pp.

[8] H. Weyl, 'Meromorphic functions and analytic curves', Annals Math. Studies, No. 12, Princeton 1943.

Department of Mathematics

National University of Singapore

Kent Ridge Crescent

Singapore 0511

Republic of Singapore 